\title{
Seven-Year Multi-Color Optical Monitoring of BL Lacertae Object S5 0716+714
}

\author{
Yan Dai \\ Department of Astronomy, Beijing Normal University, \\ Beijing 100875, China \\ Department for Popularization of Astronomy, Beijing Planetarium, \\ 138 Xizhimenwai Street, Beijing 100044, China \\ Jianghua Wu, Zong-Hong Zhu \\ Department of Astronomy, Beijing Normal University, \\ Beijing 100875, China \\ zhuzh@bnu.edu.cn \\ Xu Zhou, Jun Ma \\ Key Laboratory of Optical Astronomy, National Astronomical Observatories, \\ Chinese Academy of Sciences, 20A Datun Road, Beijing 100012, China

\section{Qirong Yuan} \\ Department of Physics and Institute of Theoretical Physics, Nanjing Normal University, \\ Nanjing 210046, China \\ and \\ Lingzhi Wang \\ Department of Astronomy, Beijing Normal University, \\ Beijing 100875, China
}

Received ; accepted 


\begin{abstract}
We have monitored the BL Lac object S5 0716+714 in five intermediate optical wavebands from 2004 September to 2011 April. Here we present the data that include 8661 measurements. It represents one of the largest databases obtained for an object at optical domain. A simple analysis of the data indicates that the object was active in most time, and intraday variability was frequently observed. In total, the object varied by 2.614 magnitudes in the $i$ band. Strong bluer-when-brighter chromatism was observed on long, intermediate, and short timescales.
\end{abstract}

Subject headings: BL lacertae Object: individual (S5 0716+714) - galaxies: active galaxies: photometry 


\section{Introduction}

Blazars constitute the most variable subclass of active galactic nuclei (AGNs). Depending on whether or not showing strong emission lines in spectra. Blazar is divided into flat-spectrum radio quasars (FSRQs) and BL Lacertae (BL Lac) objects. BL Lac objects are characterized by non-thermal continuum emission across the whole electromagnetic spectrum with absent or weak emission and absorption lines (Stickel et al. 1993), variable and high polarization (Angel \& Stockman 1980; Impey \& Neugebaur 1988; Gabuzda et al. 1989), large amplitude and rapid variability at all wavelengths from radio to gamma rays (Ravasio et al. 2002; Böttcher et al. 2003), and superluminal motion of radio components (Denn et al. 2000).

S5 $0716+714$ is a distant BL Lac. In 1979, it was discovered in a survey for sources with a $5 \mathrm{GHz}$ flux greater than $1 \mathrm{Jy}$ (Kühr et al. 1981). Because of the featureless spectrum and its strong optical polarization, it was identified as a BL Lacertae object by Biermann et al. (1981). The redshift of S5 0716+714 was uncertain until Wagner et al. (1996) estimated a value bigger than 0.3. Afterwards, Nilsson et al. (2008) acquired a deep i-band image of this object and derived a redshift of $0.31 \pm 0.08$ by using the host galaxy as a standard candle. Most recently, Danforth et al. (2012) set an upper bound of $z<0.304$ with a confidence level of $90 \%$ for this object. This source is one of the most studied BL Lac objects, because it has high brightness and strong variability. The optical duty cycle of S5 $0716+714$ is nearly unity, indicating that the source is always in an active state in the visible (Wagner \& Witzel 1995). Strong bluer-when-brighter correlations were found for both internight and intranight variations (Wu et al. 2005, 2007, 2012; Poon et al. 2009; Hao et al. 2010; Chandra et al. 2011).

This source has been intensively monitored by a number of authors. During a 4-week period of continuous monitoring, the source displayed in both optical and radio regimes a 
transition between states of fast and slow variability with a change of the typical variability timescale from about 1 to about 7 days (Quirrenbach et al. 1991). Wagner et al. (1996) investigated the rapid variations of this object in the radio, optical, ultraviolet, and X-ray regimes and found that it always keeps high amplitude change on the timescale of a few days. Sagar et al. (1999) showed an average $V-R$ color of this BL Lac to be $\sim 0.4$ mag in their one month long BVRI optical monitoring campaign in 1994. Raiteri et al. (2003) reported that the long-term optical brightness variations of this source appear to have a characteristic timescale of 3.3yr and four major optical outbursts were observed at the beginning of 1995, in late 1997, at the end of 2000, and in fall 2001. In particular, an exceptional brightening of $2.3 \mathrm{mag}$ in 9 days was detected in the $R$ band on 2000 October 30. Color analysis on the optical light curves reveals only a weak general correlation between the color index and the source brightness. Recently, Poon et al. (2009) monitored the BL Lac object S5 0716+714 in the optical band during 2008 October and December and 2009 February with a best temporal resolution of about 5 minutes in the BVRI bands. Typical timescales of microvariability range from 2 to $8 \mathrm{hr}$. The overall $V-R$ color index ranges from 0.37 to 0.59 . Strong bluer-when-brighter chromatism was found on internight timescales. The overall variability amplitude decreases with decreasing frequency.

We have monitored S5 $0716+714$ since 2004 . Here we present the data during the period from 2004 to 2011. A simple analysis is performed and the results are described.

This paper is organized as follows. The Observation and data analysis is described in Section 2. Section 3 presents the light curves. Section 4 shows the comparison result of $i$-data from us and $R$-data from other authors. The relation of color and magnitude is described in Section 5. The conclusions are given in Section 6 . 


\section{Observations and data analysis}

Our optical monitoring program of S5 $0716+714$ was carried out with the 60/90 cm Schmidt telescope located at the Xinglong Station of the National Astronomical Observatories of China (NAOC). Prior to 2006, a Ford Aerospace 2048×2048 CCD camera was mounted at its main focus. The CCD has a pixel size of $15 \mu \mathrm{m}$, and its field of view is $58^{\prime} \times 58^{\prime}$, resulting in a resolution of $1^{\prime \prime} .7$ pixel $^{-1}$. At the beginning of 2006 , the $2 \mathrm{k}$ CCD was replaced by a new $4096 \times 4096$ CCD. The field of view is now $96^{\prime} \times 96^{\prime}$, resulting in a resolution of $1^{\prime \prime} .3$ pixel $^{-1}$. The telescope is equipped with 15 intermediate-band filters, covering a wavelength range from 3000 to $10000 \AA$.

This paper includes data from 2004 September 10 to 2011 April 24. Excluding the nights with bad weather and those devoted to other targets, the actual number of nights for S5 $0716+714$ observations is 332 . We used filters in $e, i$, and $m$ bands to observe in 2004-2006, and then changed to the $c$, $i$, and $o$ bands from 2006 December. The central wavelengths of the $c, e, i, m$, and $o$ bands were 4210, 4920, 6660, 8020, and 9190 $\AA$, respectively. The central wavelength of $i$ band is similar to the $R$ band. With the observational results of stars, the magnitudes in these two bands can be transformed with $R=i+0.1$ (Zhou et al. 2003). Depending on the weather and seeing conditions, the exposure time of different bands range from 30s to 480s, and the exposures per night of different bands varies between 2 to 43 .

The data reduction procedure includes bias subtraction, flat-fielding, extraction of instrumental aperture magnitude, and flux calibration. We used differential photometry. For each frame, the instrumental magnitudes of the blazar and four comparison stars (See Fig.1) were extracted at first. The radii of the aperture and the sky annuli were adopted as 3, 7, and 10 pixels, respectively. Then the brightness of the blazar was measured relative to the average brightness of the three reference stars 3, 4, and 5. Star 6 acted as a check 
star, which has an apparently similar brightness as the blazar (for a reasonable selection of reference and check stars, see Howell et al. 1988). The differential magnitude of star 6 is the difference between the magnitude of star 6 and the average magnitude of star 4 and 5, so as to verify the stable fluxes of the four comparison stars, and to verify the accuracy of our measurements. The $c, e, i, m$, and $o$ magnitudes of the 4 comparison stars were obtained by observing them and the standard star HD 19945 on a photometric night and are listed in Table 1.

\section{Light Curves}

The samples of observational log and results are given in Tables 2-6. The columns are observation date and time in universal time, Julian date, exposure time in second, magnitude and error of S5 0716+714, and differential magnitude of star 6 (its nightly averages were set to zero). Figure 2 shows the light curves of the overall monitoring period in the five bands.

The source remained active during the whole monitoring period. The variation amplitudes of $e, i$, and $m$ bands from 2004 September 10 to 2006 March 29 are 1.200, 1.156, and 1.127 mags, respectively, and the variation amplitudes of $c, i$, and $o$ bands from 2006 December 6 to 2011 April 24 are 2.763, 2.614, and 2.522 mags, respectively. The amplitude of variation tends to decrease with decreasing frequency. The light curves keep fluctuating during the whole monitoring period. The curves get the extreme bright value on 2004 September 10 (JD 2,453,259), 2007 October 20 (JD 2,454,394), 2008 April 22 (JD 2,454,579), and 2010 September 28 (JD 2,455,468), and get the extreme dark value on 2005 January 29 (JD 2,453,400), 2007 December 16 (JD 2,454,451), and 2011 April 24 (JD 2,455,676). A faintest optical state was recorded on 2007 December 16 (JD 2,454,451), . Nilsson et al. (2008) acquired a deep i-band image of this object at that state and derived 
a redshift of $0.31 \pm 0.08$ by using the host galaxy as a 'standard candle'.

The BL Lac object S5 $0716+714$ is one of the brightest BL Lac objects noted for its microvariability. In order to confirm whether or not the object was variable in one day, a quantitative assessment was carried out. For each of the 233 nights with observational duration longer than 2 hours, we used a chi-square inspection to check whether there is intraday variability (IDV) (Penston \& Cannon 1970; Kesteven et al. 1976; de Diego 2010). The chi-square value was compared with the critical value at the $95 \%$ confidence level. If the former was greater than the latter, the null hypothesis that there was no variability was rejected. As a result, 138 nights (62\% of 233 ) with IDV were identified. The observed fastest variation of S5 $0716+714$ varied by 0.117 mags in 1.1 hrs in the $c$ band on 2011 March 5 (JD 2,455,626), as indicated by the bottom line with arrows in Figure 3. The similar work was made by Villata et al. (2000). They found that the source exhibited strong variability with similar trend but different amplitudes in all bands, and noted the monotonic brightness increase in $B$ band for about 130 minutes. The steepest (linear) part has a rising rate of $0.002 \mathrm{mag}$ per minute and a duration of about 45 minutes.

\section{Compare with Other Data}

In Section 2, a transforming formula between the $i$ and $R$ magnitudes was mentioned.

This formula was derived from the observational result of stars (Zhou et al. 2003). However, the spectral shape of blazars is quite different from that of stars. So the transforming formula may be different for blazars. Therefore, we made a comparison between our $i$-band data and the $R$-band data of other authors in order to find an empirical relation between them. Villata et al. (2000) and Poon et al. (2009) have made intensive monitoring on the same object. Their data were adopted and matched in time with ours with a threshold of less than 0.02 days (or 28.8 minutes). As the results, $24 R$-i matches were found for Villata 
et al. and our data, and $97 R-i$ matches were found for Poon et al. and our data. The average time differences are 0.0038 and 0.0009 days for the 20 and 97 matches, respectively. Two $R-i$ diagrams were plotted in the top and middle panels of Figure 4. Two linear regressions give the transforming formulae as $R=(0.964 \pm 0.015) \times i+(0.279 \pm 0.187)$ and $R=(0.976 \pm 0.009) \times i+(0.059 \pm 0.123)$, respectively. If all matches are plotted together, as shown in the bottom panel of Figure 4, the linear regression gives the formula as $R=(0.897 \pm 0.004) \times i+(1.127 \pm 0.048)$.

\section{Relation of Color and Magnitude}

The long-term color behavior of S5 0716+714 was studied based on our data. For the 2004-2006 data, the $e-m$ color was calculated and plotted vs. the $e$ magnitude in the top panel of Figure 5. For the data after 2006 December, the $c-o$ color was calculated and plotted vs. the $c$ magnitude in the central panel of Figure 5. The bottom panels illustrate how the $c-o$ color and $c$ magnitude changed with time. Despite the discontinuity in the top panel and significant scatter in the central panel, there is an overall bluer-when-brighter chromatism in both panels.

In order to investigate the color behavior of S5 $0716+714$ on the intermediate timescale, three episodes in our monitoring are isolated. They are from JDs 2,454,101 to 2,454,115, from JDs 2,454,429 to 2,454,463, and from JDs 2,455,597 to 2,455,629. These three episodes lasted from two weeks to more than one month. In these periods, we have relatively continuous monitoring, and the object showed significant variations. The light curves and the corresponding color-magnitude diagrams are plotted in Figure 6 for the three episodes. There are strong color-magnitude correlations. The correlation coefficients are 0.89, 0.78, and 0.82 , respectively. The bluer-when-brighter chromatism on intermediate timescale is found by other authors (e.g., Villata et al. 2000; Wu et al. 2007). On intraday timescales, 
the object also displays strong bluer-when-brighter chromatism. Some examples are given in Figure 7.

Depending on the balance between escape, acceleration, and cooling of the electrons with different energy, either soft (low energy) or hard (high energy) lags are expected (Kirk et al. 1998). This will lead to a loop-like path of the blazars state in a colormagnitude (or spectral index-flux) diagram. The direction of this spectral hysteresis can be either clockwise or anticlockwise. It depends on the relative position between the observing frequency and the peak frequency of the synchrotron component in the SED of the blazar as well as the relative values of the acceleration, cooling and escape timescales (Chiaberge \& Ghisellini 1999; Dermer 1999).

In our monitoring, we got an inconspicuous loop flare in internight timescale and an obvious loop flare in intranight timescale, which are shown in Figure 8. The upper-left panel is the light curve of JDs 2,454,390 2,454,398, which shows a flare in cio bands. The corresponding color-magnitude diagram is displayed in the upper-right panel, in which the numbers denote time sequence. There were not significant variations during these day, so we averaged the data in day to decrease system error. The points spread as diagonal distribution in color-magnitude diagram. The loop flare is far less obvious. The result of intranight timescale, JD 2,455,621, is shown in the bottom of Figure 7 . The numbers denote the time sequence. An anticlockwise loop can be seen in the lower-right panel. As Kirk et al. (1998) supposed, if the loop is traced anticlockwise, there might be a flare propagating from lower to higher energy, as particles are gradually accelerated into the radiating window. The frequency of $c$ and $o$ band are $7.13 \times 10^{14} \mathrm{~Hz}$ and $3.26 \times 10^{14} \mathrm{~Hz}$. In 1999, Giommi et al. found that the frequency is between $10^{14}$ and $10^{15} \mathrm{~Hz}$. Our anticlockwise loop imply that the peak frequency of short band in the SED of the blazar should be higher than $7.13 \times 10^{14} \mathrm{~Hz}$. 


\section{Conclusions}

We have monitored the BL Lac object S5 $0716+714$ in five intermediate optical wavebands from 2004 September to 2011 April by the 60/90 cm Schmidt telescope located at the Xinglong Station of the National Astronomical Observatories of China (NAOC). We collected 8661 data points with error less than 0.05 mags. It represents one of the largest databases obtained for an object at optical domain and can be used to study both the longand short-term flux and spectral variability of this object. It can also be correlated with the data in the radio, X-ray, or gamma-ray wavelengths in order to investigate the broad-band behavior of this object. A simple analysis of the data indicates that the object was active in most time. The overall amplitudes of $e, i$, and $m$ bands from 2004 September 10 to 2006 March 29 are 1.200, 1.156, and 1.127 mags, respectively, and the overall amplitudes of $c$, $i$, and $o$ bands from 2006 December 6 to 2011 April 24 are 2.763, 2.592, and 2.522 mags, respectively. The amplitude of variation tends to decrease with decreasing frequency. The bluer-when-brighter phenomenon is effectively confirmed on long, intermediate, and short timescales. It is an important support to the shock-in-jet model in which shocks propagate down the relativistic jet, accelerating particles and/or compressing magnetic fields, leading to the observed flux and spectral variability (Marscher \& Gear 1985; Qian et al. 1991). There were 138 nights of IDV captured during the whole monitoring period.

The authors thank the anonymous referee for constructive suggestions and insightful comments. We thank Villata, M. for kindly sending us the WEBT data on S5 0716+714. This work has been supported by Chinese National Natural Science Foundation grants 11273006, 11173016, and 11073023. WJH is supported by National Basic Research Program of China 973 Program 2013CB834900. ZHZ acknowledges that this work is supported by the Ministry of Science and Technology National Basic Science Program (Project 973) under Grant No.2012CB821804, the Fundamental Research Funds for the Central Universities 
and Scientific Research Foundation of Beijing Normal University. 


\section{REFERENCES}

Angel, J. R. P., Stockman, H. S. 1980, ARA\&A, 18, 321

Biermann, P.L., et al. 1981, ApJ, 247, 53

Böttcher, M. et al. 2003, ApJ, 596, 847

Chandra, S., Baliyan, K. S., Ganesh, S., \& Joshi, U. C. 2011, ApJ, 731, 118

Chiaberge, M. \& Ghisellini, G. 1999, MNRAS, 306, 551

Danforth, C. W., Nalewajko, K., France, K., \& Keeney, B. A. 2012, submitted to ApJ, arXiv:1209.3325

Denn, G. R., Mutel, L. R., Marscher, A. P. 2000, ApJS, 129, 61

Dermer, C. D. 1998, ApJ, 501, 157

de Diego, J. A. 2010, AJ, 139, 1269

Fukugita, M., et al. 1996, AJ, 111, 1748

Gabuzda, D. C., Cawthorne, T. V., Roberts, D. H., \& Wardle, J. F. C. 1989, ApJ, 347, 701

Hao, J., Wang, B., Jiang, Z., \& Dai, B. 2010, RAA, 10, 125

Howell, S. B., Mitchell, K. J., \& Warnock, A. 1998, AJ, 95, 247

Kesteven, M. J. L., Bridle, A. H., \& Brandie, G. W. 1976, AJ, 81, 919

Impey, C. D., \& Neugebaur, G. 1988, AJ, 95, 307

Kirk, J. G., Rieger, F. M., \& Mastichiadis, A. 1998, A\&A, 333, 452

Kühr, H., Witzel, A., Pauliny-Toth, I. I. K., \& Nauber, U. 1981, A\&AS, 45, 367 
Marscher, A. P., \& Gear, W. K. 1985, ApJ, 298, 114

Nilsson, K., Pursimo, T., Sillanpää, A., Takalo, L. O., \& Lindfors, E. 2008, A\&A, 487, 29

Oke, J. B. \& Gunn, J. E. 1983, ApJ, 266, 713

Penston, M. V., \& Cannon, R. D. 1970, Royal Greenwich Obs. Bull., 159, 83

Poon, H., Fan, J. H., \& Fu, J. N. 2009, ApJS, 185, 511

Qian, S. J., Quirrenbach, A., Witzel, A., Krichbaum, T. P., Hummel, C. A., \& Zensus, J. A. 1991, A\&A, 241, 15

Quirrenbach, A., et al. 1991, ApJ, 372, 71

Raiteri, C. M., et al. 2003, A\&A, 402, 151

Ravasio, M. et al. 2002, A\&A, 383, 763

Sagar, R., Gopal-Krishna, Mohan, V., Pandey, A. K., Bhatt, B. C., \& Wagner, S. J. 1999, A\&AS, 134, 453

Stalin, C. S., Gopal-Krishna, Sagar, R., Wiita, P. J., Mohan, V., \& Pandey, A. K. 2006, MNRAS, 366, 1337

Stickel, M., Fried, J. W., \& Kühr, H. 1993, A\&AS, 98, 393

Vagnetti, F., Trevese, D., \& Nesci, R. 2003, ApJ, 590, 123

Villata, M., et al. 2000, A\&A, 363, 108

Wagner, S. \& Witzel, A. 1995, ARA\&A, 33, 163

Wagner, S. J., et al. 1996, AJ, 111, 2187

Wu, J., et al. 2005, AJ, 129, 1818 
Wu, J., et al. 2007, AJ, 133, 1599

Wu, J., et al. 2012, AJ, 143, 108

Yan, H., et al. 2000, PASP, 112, 691

Zhou, X., et al. 2003, A\&A, 397, 361 


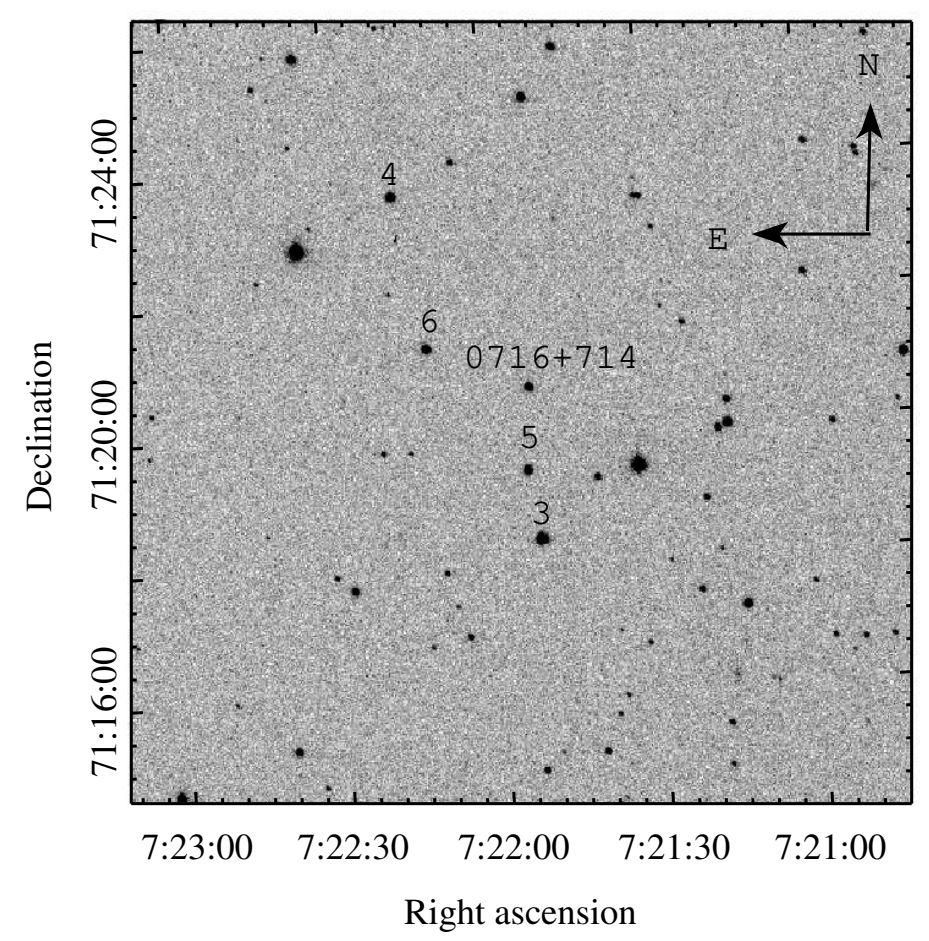

Fig. 1.- Finding chart of S5 $0716+714$ and the 4 comparison stars taken with the $60 / 90$ Schmidt telescope and filter $i$ on 2011 April 12 (JD 2,455,664). The size is $12^{\prime} \times 12^{\prime}$ (or $512 \times 512$ in pixels). 

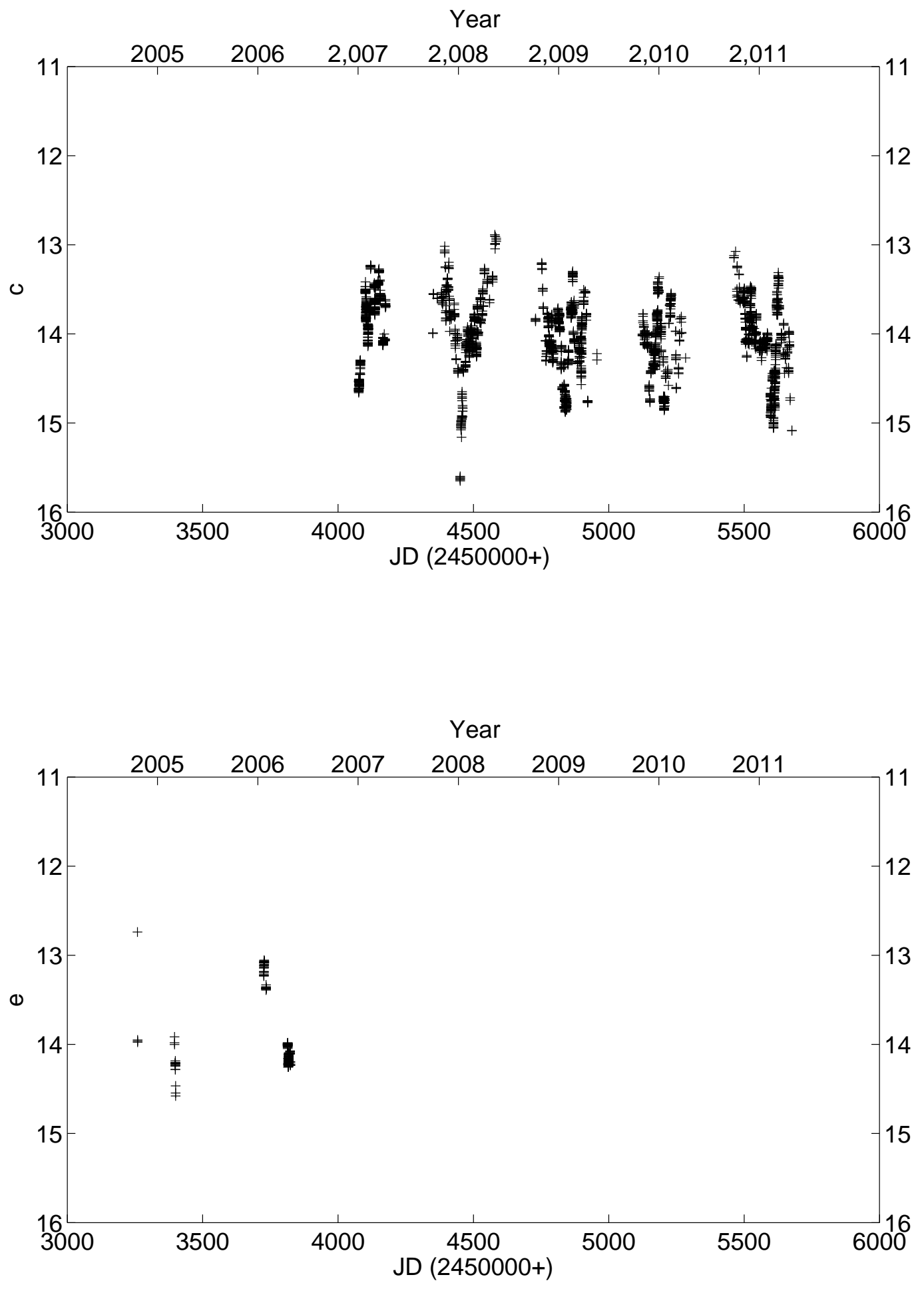

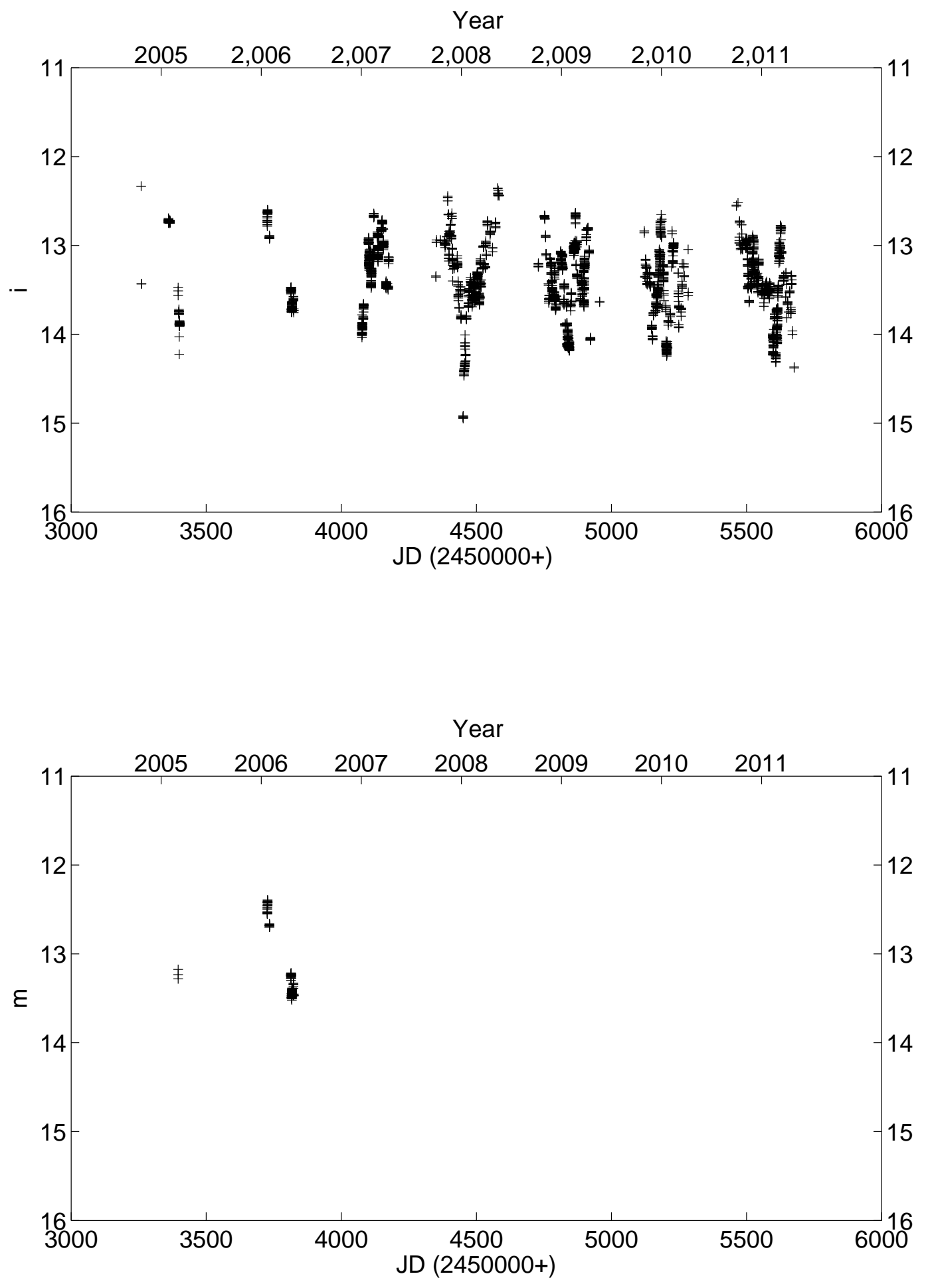


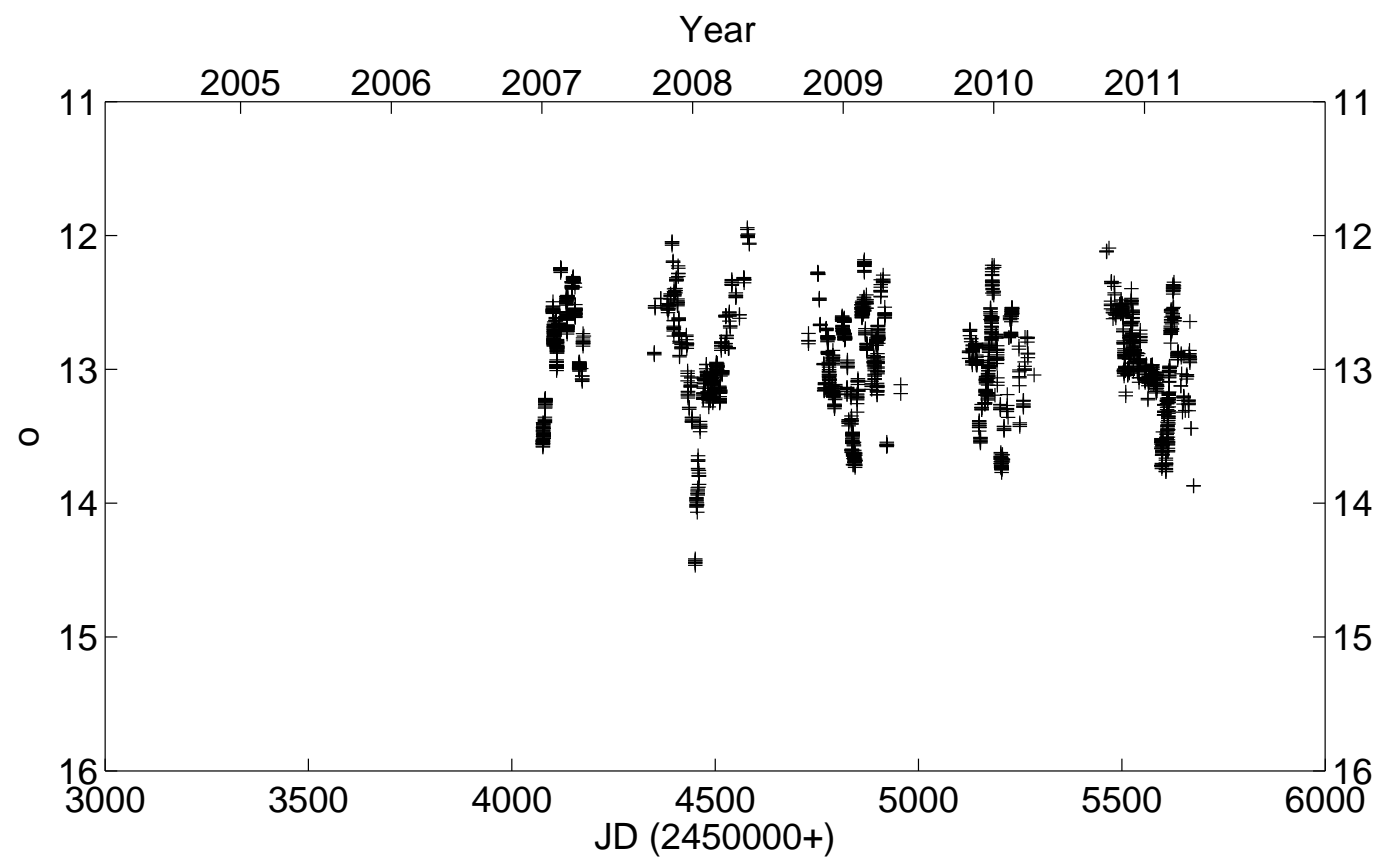

Fig. 2.- Light curves of S5 0716+714 in the $c, e, i, m$, and $o$ bands. 


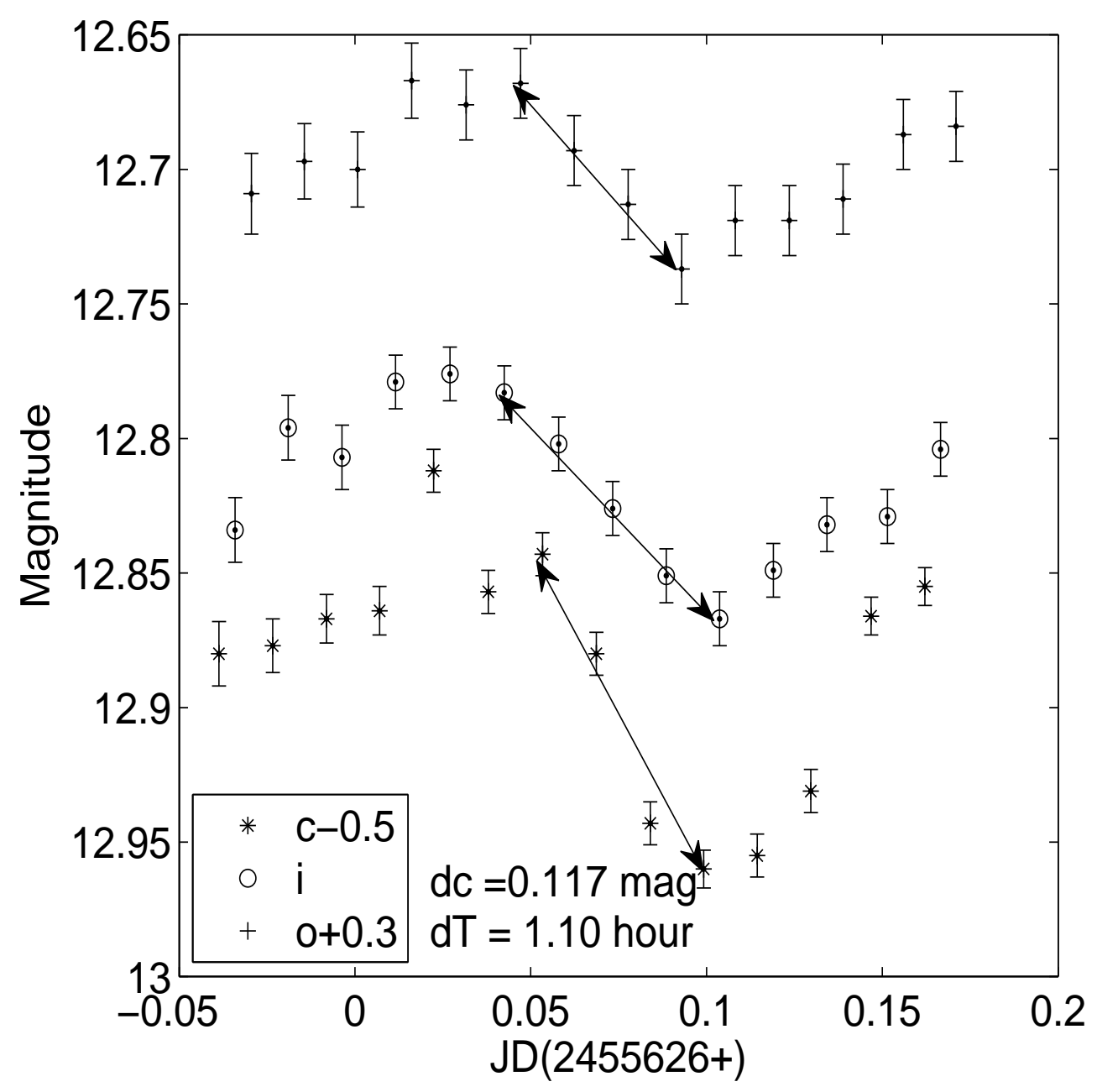

Fig. 3.- The observed fastest variation of S5 $0716+714$. The object varied by 0.117 mags in $1.1 \mathrm{hrs}$ in the $c$ band, as indicated by the bottom line with arrows. 

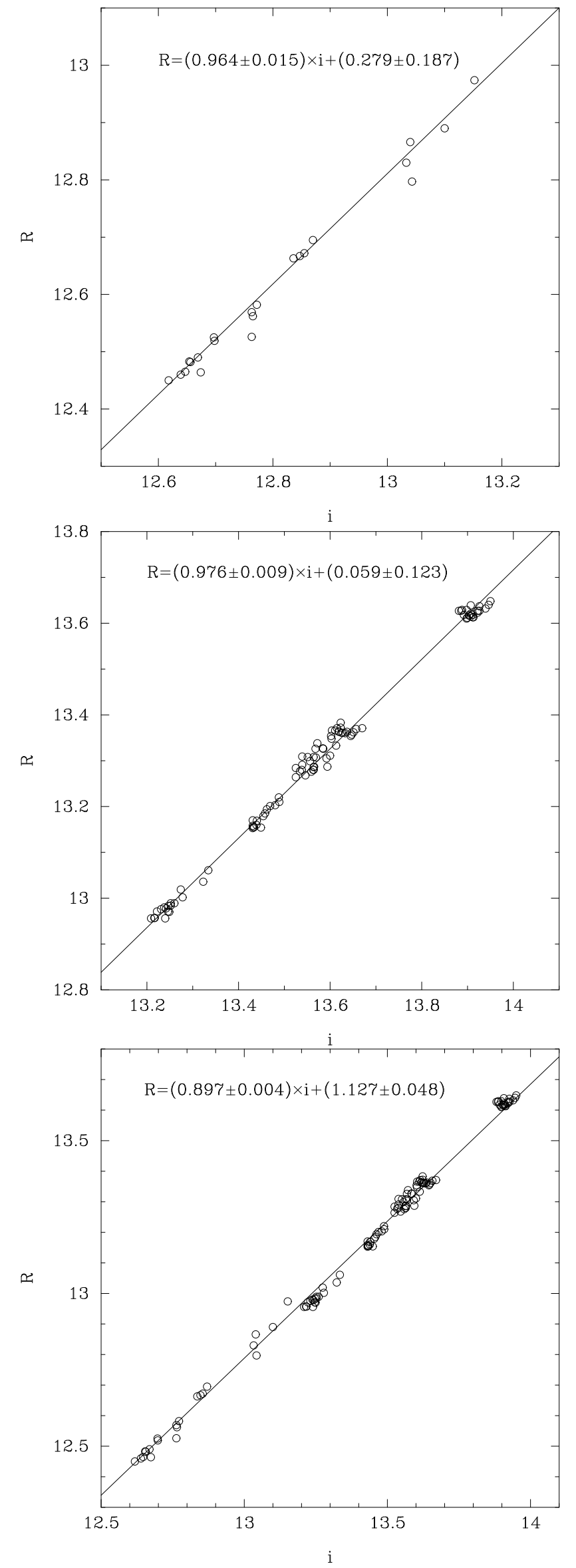



Fig. 5.- Color-magnitude diagrams of $\mathrm{em}$ bands (top) and co bands (middle) and the color 

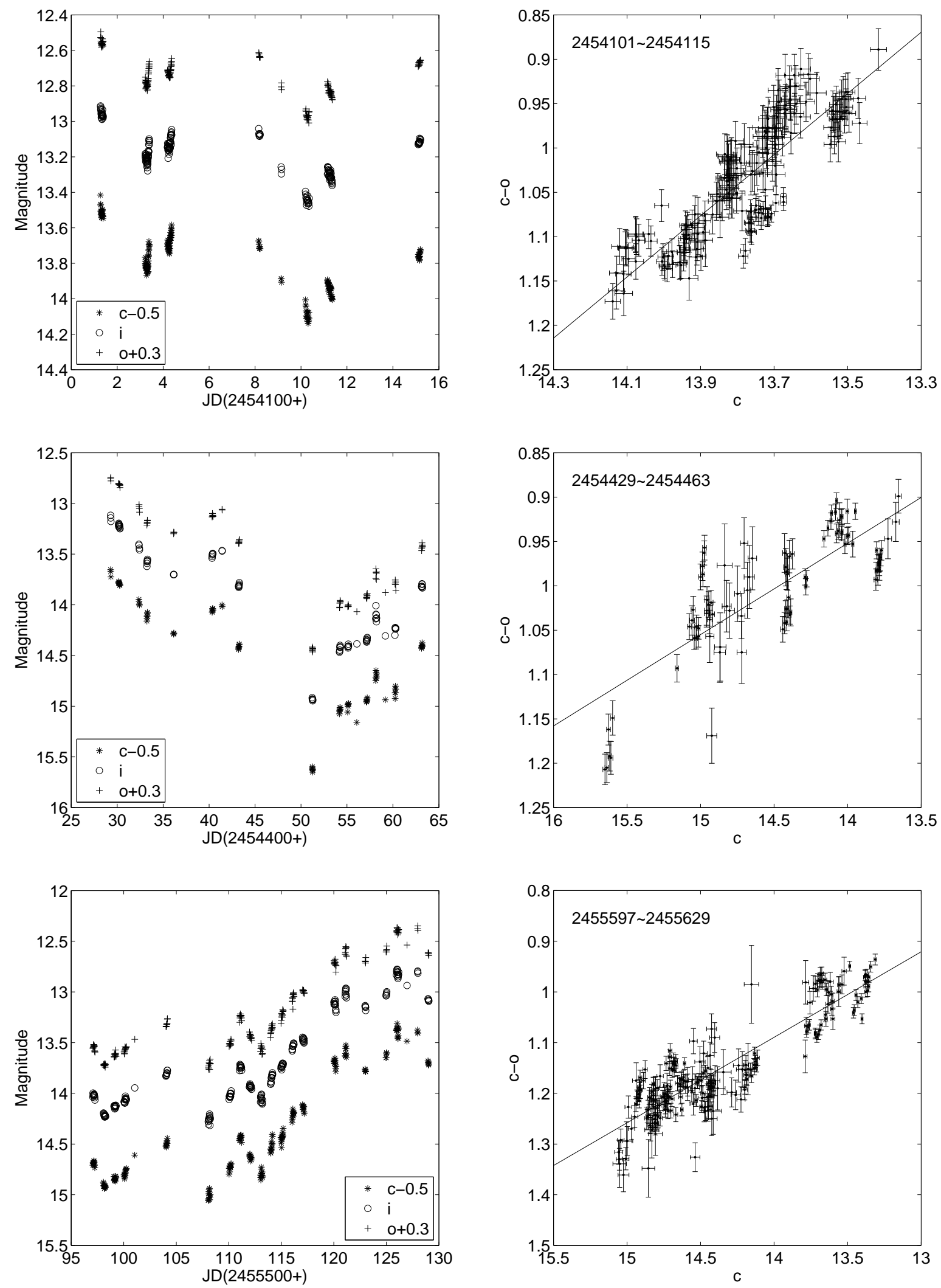

Fig. 6.- Light curves (left) and Color-magnitude diagrams (right) of intermediate timescales. The line in the right figures is the linear fit to the data. 

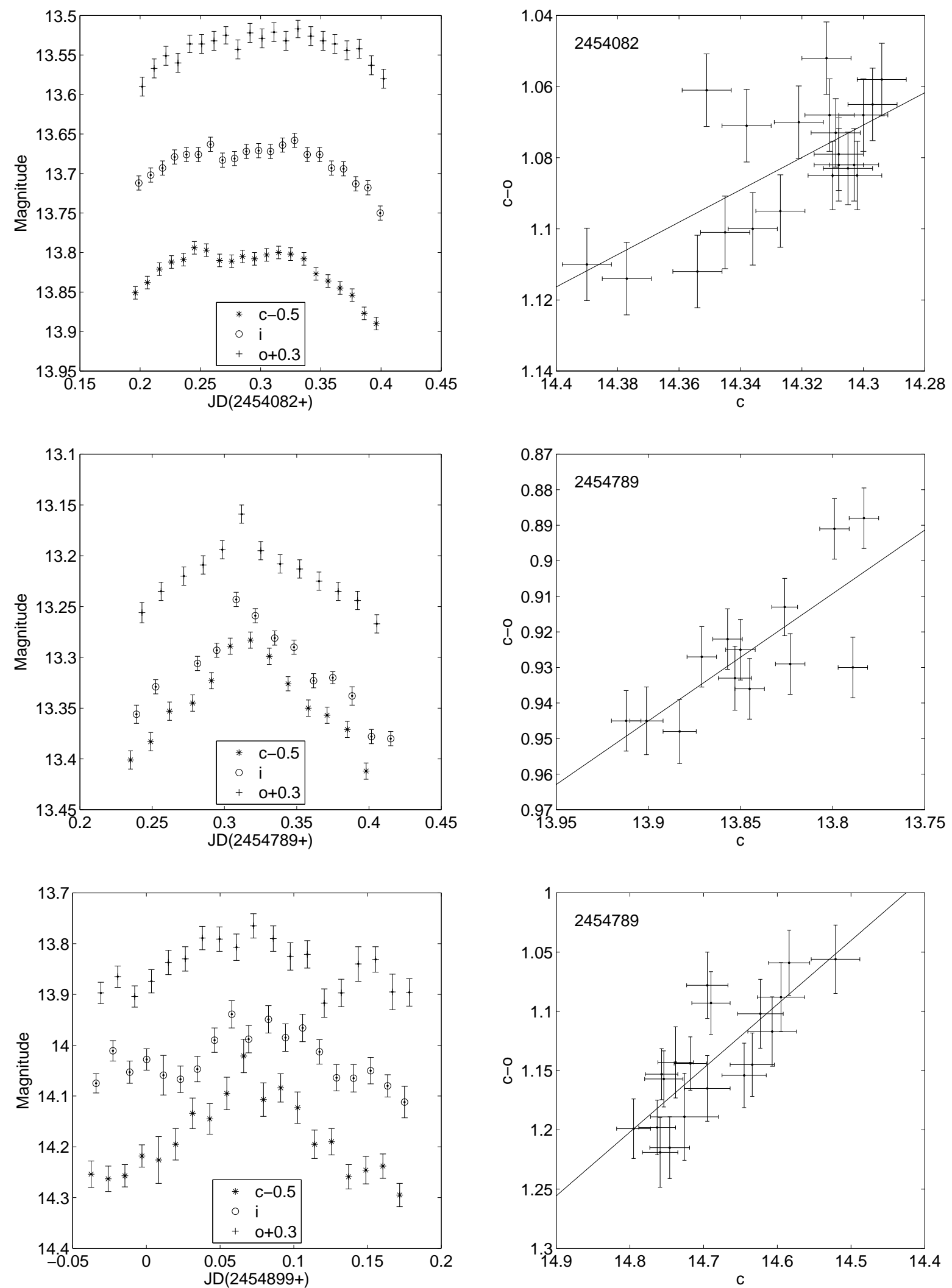

Fig. 7.- Light curves (left) and Color-magnitude diagrams (right) of IDV data. The line in the right figures is the linear fit to the data. 

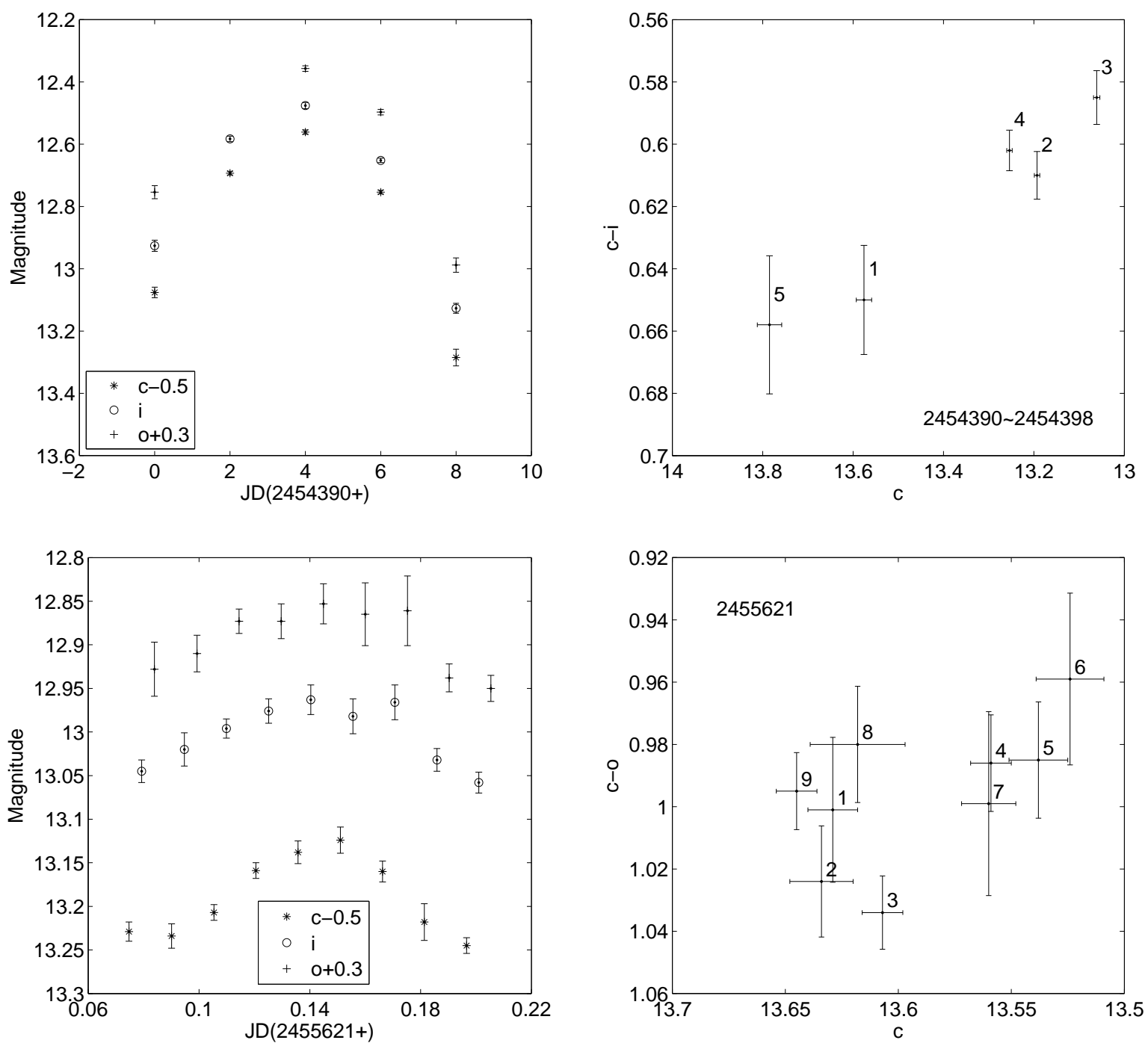

Fig. 8.- Light curves (left) and color-magnitude diagrams (right) for the internight (upper) and intranight (lower) timescales. The numbers in the right panels denote the time sequence. 
Table 1. Magnitudes of 4 Comparison Stars.

\begin{tabular}{cccccc}
\hline \hline Star & $c$ & $e$ & $i$ & $m$ & $o$ \\
\hline & & & & & \\
3 & 13.033 & 12.706 & 12.271 & 12.190 & 12.178 \\
4 & 13.574 & 13.412 & 13.098 & 13.050 & 13.060 \\
5 & 14.126 & 13.794 & 13.363 & 13.264 & 13.223 \\
6 & 14.195 & 13.890 & 13.442 & 13.370 & 13.329 \\
\hline
\end{tabular}

Table 2. Data of $c$ band

\begin{tabular}{ccccccc}
\hline \hline & & & & & & \\
Date(UT) & Time & Julian Date & $\operatorname{Exp}$ & $c$ & $c_{\text {err }}$ & dfmag \\
\hline & & & & & & \\
20061206 & $18: 43: 43.0$ & 2454076.28036 & 300 & 14.649 & 0.016 & 0.002 \\
20061206 & $18: 58: 33.0$ & 2454076.29066 & 300 & 14.636 & 0.020 & -0.018 \\
20061206 & $19: 12: 43.0$ & 2454076.30050 & 300 & 14.639 & 0.014 & 0.011 \\
20061206 & $19: 27: 06.0$ & 2454076.31049 & 300 & 14.660 & 0.014 & 0.004 \\
20061206 & $19: 41: 42.0$ & 2454076.32063 & 300 & 14.612 & 0.014 & -0.003 \\
\hline
\end{tabular}

Note. - Table 2 is published in its entirety in the electronic edition of the The Astrophysical Journal Supplement. A portion is shown here for guidance regarding its form and content. 
Table 3. Data of $e$ band

\begin{tabular}{lcccccc}
\hline \hline \multirow{2}{*}{ Date(UT) } & Time & Julian Date & Exp & $e$ & $e_{\text {err }}$ & dfmag \\
\hline 20050128 & $15: 57: 45.0$ & 2453399.16510 & 300 & 14.216 & 0.027 & -0.009 \\
20050128 & $16: 07: 44.0$ & 2453399.17204 & 300 & 14.212 & 0.026 & -0.028 \\
20050128 & $16: 17: 43.0$ & 2453399.17897 & 300 & 14.186 & 0.023 & 0.015 \\
20050128 & $16: 27: 57.0$ & 2453399.18608 & 300 & 14.239 & 0.029 & -0.015 \\
20050128 & $16: 37: 53.0$ & 2453399.19297 & 300 & 14.242 & 0.026 & 0.011 \\
\hline
\end{tabular}

Note. - Table 3 is published in its entirety in the electronic edition of the The Astrophysical Journal Supplement. A portion is shown here for guidance regarding its form and content. 
Table 4. Data of $i$ band

\begin{tabular}{lcccccr}
\hline \hline \multirow{2}{*}{ Date(UT) } & Time & Julian Date & Exp & $i$ & $i_{\text {err }}$ & dfmag \\
\hline 20041220 & $17: 45: 00.0$ & 2453360.23958 & 240 & 12.739 & 0.011 & -0.012 \\
20041220 & $17: 51: 11.0$ & 2453360.24388 & 240 & 12.745 & 0.010 & 0.010 \\
20041220 & $17: 55: 40.0$ & 2453360.24699 & 240 & 12.717 & 0.010 & -0.026 \\
20041220 & $18: 00: 06.0$ & 2453360.25007 & 240 & 12.748 & 0.011 & 0.032 \\
20041220 & $18: 04: 36.0$ & 2453360.25319 & 240 & 12.733 & 0.014 & -0.008 \\
\hline
\end{tabular}

Note. - Table 4 is published in its entirety in the electronic edition of the The Astrophysical Journal Supplement. A portion is shown here for guidance regarding its form and content. 
Table 5. Data of $m$ band

\begin{tabular}{ccccccc}
\hline \hline \multirow{2}{*}{ Date(UT) } & Time & Julian Date & Exp & $m$ & $m_{\text {err }}$ & dfmag \\
\hline 20051221 & $18: 46: 03.0$ & 2453726.28198 & 300 & 12.549 & 0.007 & -0.019 \\
20051221 & $19: 02: 20.0$ & 2453726.29329 & 300 & 12.550 & 0.007 & -0.013 \\
20051221 & $19: 18: 14.0$ & 2453726.30433 & 300 & 12.551 & 0.007 & 0.012 \\
20051221 & $19: 34: 46.0$ & 2453726.31581 & 300 & 12.545 & 0.008 & 0.007 \\
20051221 & $19: 50: 41.0$ & 2453726.32686 & 300 & 12.537 & 0.008 & -0.002 \\
\hline
\end{tabular}

Note. - Table 5 is published in its entirety in the electronic edition of the The Astrophysical Journal Supplement. A portion is shown here for guidance regarding its form and content. 
Table 6. Data of $o$ band

\begin{tabular}{ccccccr}
\hline \hline \multirow{2}{*}{ Date(UT) } & Time & Julian Date & Exp & $o$ & $o_{\text {err }}$ & dfmag \\
\hline 20061206 & $18: 51: 49.0$ & 2454076.28598 & 300 & 13.580 & 0.018 & 0.004 \\
20061206 & $19: 06: 42.0$ & 2454076.29632 & 300 & 13.555 & 0.017 & 0.007 \\
20061206 & $19: 21: 04.0$ & 2454076.30630 & 300 & 13.557 & 0.016 & 0.008 \\
20061206 & $19: 35: 38.0$ & 2454076.31641 & 300 & 13.562 & 0.016 & -0.005 \\
20061206 & $19: 50: 16.0$ & 2454076.32657 & 300 & 13.532 & 0.016 & 0.004 \\
\hline
\end{tabular}

Note. - Table 6 is published in its entirety in the electronic edition of the The Astrophysical Journal Supplement. A portion is shown here for guidance regarding its form and content. 